\title{
Application of the Riccati Method to the adiabatic oscillations of stars
}

\section{Takata}

Department of Astronomy, School of Science, The University of Tokyo, Bunkyo-ku, Tokyo 113-0033, Japan

W. Löffler ${ }^{1}$

Astronomical Institute, Graduate School of Science, Tohoku University, Aramaki, Aoba, Sendai 980-8578, Japan

\begin{abstract}
The eigenmodes of the adiabatic oscillations of stars are usually calculated numerically by solving the system of the four linear first-order differential equations using either the relaxation method or the shooting method. Finding some shortcomings in these conventional methods, we adopt another method, namely the Riccati method, in which it is not the system of the linear differential equations but the nonlinear matrix Riccati equation that is solved numerically. After describing the method, we discuss its advantages and give some demonstrations.
\end{abstract}

\section{Introduction}

In stellar oscillation problems, it is necessary to calculate a variety of eigenmodes of stellar models whose radial and angular orders generally range from very low to very high values. This is particularly true when we perform numerical inversions for the solar structure, in which a large number of modes must be calculated very accurately. Conventionally the relaxation method is used to solve this problem (cf. Christensen-Dalsgaard \& Mullan 1994). In order to get reliable answers by this method, we need, however, to invoke various techniques like adjusting the mesh allocation to achieve the desired accuracy or truncating the mesh points where the eigenfunctions become extremely small, typically in the central region of the star. For these reasons the relaxation method is rather tedious and troublesome to implement.

As an alternative, we adopt the Riccati method, in which systems of linear equations are transformed into a nonlinear matrix Riccati equation. The dependent variable of this new equation does not retain the dependent variables of the original linear equations themselves, but keeps the relations among them. Note that this method has already been used in the case of nonadiabatic oscillations of stars (Gautschy \& Glatzel 1990a,b; Glatzel \& Gautschy 1992).

\footnotetext{
${ }^{1}$ Present address: Astronomisches Institut der Universität Basel, Venusstrasse 7, CH 4102 Binningen, Switzerland
} 


\section{Riccati method applied to the linear adiabatic oscillations of stars}

The system of equations for the linear adiabatic oscillations of stars is composed of four linear first-order differential equations with four dependent variables as well as two linear boundary conditions each at the center and the surface (e.g. Unno et al. 1989). We show in the following that this system can be transformed to a nonlinear $2 \times 2$ matrix differential equation with the appropriate boundary conditions.

Since the order of the original system of differential equations is four, there exist four linearly independent solutions. From these we can construct at most two linearly independent solutions that satisfy the two central boundary conditions. Let us denote these solutions by $\boldsymbol{y}^{(1)}$ and $\boldsymbol{y}^{(2)}$, where $\boldsymbol{y}=\left(y_{1} y_{2} y_{3} y_{4}\right)^{T}$ is a four-component vector. Suppose that we decompose these vectors as $\boldsymbol{y}^{(i)}=$ $\left(\boldsymbol{u}^{(i)^{T}} \boldsymbol{v}^{(i)^{T}}\right)^{T}(i=1,2)$, where $\boldsymbol{u}^{(i)}=\left(y_{1} y_{2}\right)^{T}$ and $\boldsymbol{v}^{(i)}=\left(y_{3} y_{4}\right)^{T}$ are twocomponent vectors each containing two components of $y^{(i)}$. Then we can define a $2 \times 2$ matrix $R$ by the following relation:

$$
R \equiv\left(\begin{array}{ll}
\boldsymbol{u}^{(1)} & \boldsymbol{u}^{(2)}
\end{array}\right)\left(\begin{array}{ll}
\boldsymbol{v}^{(1)} & \boldsymbol{v}^{(2)}
\end{array}\right)^{-1} .
$$

Using the original system of the four differential equations, we can show that this matrix $R$ satisfies the differential equation,

$$
x \frac{d R}{d x}=B+A R-R D-R C R,
$$

where $x$ is the fractional radius. $A, B, C$, and $D$ are the $2 \times 2$ submatrices of the $4 \times 4$ coefficient matrix of the original system of differential equations, which result from the decomposition of $\boldsymbol{y}^{(i)}$.

In the same manner we can derive a second matrix similar to the one given by Eq. (1) based on the two independent solutions satisfying the outer boundary conditions. This second matrix obeys the same type of matrix equation as given in Eq. (2).

The boundary values of the matrix $R$ are directly derived from the boundary conditions of the original system and contain at most one free parameter corresponding to the eigenvalue $\omega^{2}$. Assuming a trial value for $\omega^{2}$, we can start the integration of Eq. (2) outward from the center and inward from the surface.

The outwards and inwards integrated solutions should be matched at a certain point (fitting point) between the center and the surface. If we denote the results of the outwards and inwards integrations by $R_{0}$ and $R_{\mathrm{i}}$, respectively, it can be shown that the matching condition at the fitting point is given by $\operatorname{det}\left(R_{\mathrm{i}}-R_{\mathrm{o}}\right)=0$, which determines the eigenvalue $\omega^{2}$. Thus, this method is a kind of shooting method. A more detailed description of this so-called Riccati method can be found in, e.g., Ascher et al. (1995).

There exists a problem if the inverse of the matrix $\left(\boldsymbol{v}^{(1)} \boldsymbol{v}^{(2)}\right)$ in Eq. (1) does not exist. In this case, we can always redefine the vectors $u$ and $v$ by rearranging the components of $y$ in such a manner that $\left(\boldsymbol{v}^{(1)} \boldsymbol{v}^{(2)}\right)$ becomes regular. This procedure of re-imbedding therefore guarantees that the Riccati matrices can always be defined. 
After solving the matrix Riccati Eq. (2), the eigenfunctions can be computed by integrating

$$
x \frac{d \boldsymbol{v}}{d x}=(C R+D) \boldsymbol{v} \quad \text { and } \quad \boldsymbol{u}=R \boldsymbol{v}
$$

with the initial value given at the fitting point as the solution of the equation $\left(R_{\mathrm{i}}-R_{0}\right) \boldsymbol{v}=\mathbf{0}$. Note that we have to solve Eq. (3) twice, once from the fitting point inwards to the center and once from the fitting point outwards to the surface.

\section{Advantages of the Riccati method}

The Riccati method is particularly useful when solving the eigenproblem at very high radial orders or spherical degrees, where the amplitudes of the eigenfunctions vary strongly over the star. In such a case, the relaxation method suffers from the drawback that it can no longer resolve the very small eigenfunction amplitudes deep inside the evanescent region (typically near the center of the star), whereas the shooting method tends to become numerically unstable due to the high amplitude contrast between the boundary conditions and the fitting point.

Although there exist remedies for these insufficiencies of the conventional methods, such as, for example, the mesh truncation or the rescaling of the amplitude, we do not have to rely on such special techniques when using the Riccati method. The fact that the Riccati method can be applied without these special provisions irrespective of radial orders or angular degrees makes it very efficient, especially in the case where we have to compute a large variety of wildly different eigenmodes in a single stellar model, e.g. in the numerical inversion problem in helioseismology.

We regard this kind of efficiency as the main advantage of the adiabatic Riccati method.

\section{Numerical demonstrations and discussion}

To demonstrate the numerical precision of the Riccati method, we list the eigenfrequencies of selected modes of an $n=3$ polytrope with eight significant digits (Table 1). Switching from double to quadruple precision shows that our polytropic eigenfrequencies are numerically stable, not only in this case but also for $n=0, n=1.5$, and $n=4$.

It may be argued that this kind of high accuracy in the eigenfrequencies is meaningless because there probably exist much larger uncertainties in the stellar equilibrium models on which these frequencies are based. This is in fact true in the forward-problem, where observed eigenfrequencies are compared to theoretical ones based on the stellar models.

However, if we consider the inverse problem where the observed frequencies are inverted for the stellar structure, matters are different. In this case the theoretical frequencies based on stellar models are at most used as references so that the inverted stellar structure is actually free from uncertainties in the stellar models on which the theoretical frequencies were based. Therefore, in the 
Table 1. Dimensionless squared eigenfrequencies of a polytrope of index 3 calculated by the Riccati method.

\begin{tabular}{rcccc}
\hline \multicolumn{1}{c}{$n$} & $l=0$ & $l=1$ & \multicolumn{1}{c}{$l=4$} & $l=100$ \\
\hline-40 & - & 0.0092875187 & 0.086259638 & 6.1558068 \\
-20 & - & 0.034113781 & 0.29486627 & 8.1522986 \\
-1 & - & 2.5159280 & 8.0029627 & 14.068287 \\
0 & - & - & 10.052910 & 104.54425 \\
1 & 9.2547061 & 11.404375 & 20.752029 & 208.93919 \\
20 & 711.95699 & 743.47351 & 829.68797 & 2692.8889 \\
40 & 2656.3155 & 2717.9701 & 2891.9866 & 6695.2075 \\
\hline
\end{tabular}

inverse-problem approach, the theoretical frequencies have to be more accurate than the observed frequencies, which often have smaller errors than the stellar models. We believe that the Riccati method described in this paper is a suitable tool to accomplish this task.

We finally note that the Riccati method is not only useful for numerical calculations but also for the analytical investigation of boundary value problems, since it gives us a completely different point of view by treating the differential equation and the boundary conditions inseparably. This characteristic of the method could help to elucidate the mathematical structure of the linear adiabatic oscillations of stars. The possible problems that could be attacked using the Riccati method include mode classification without assuming the Cowling approximation (cf. Lopes 2001) and the nondegeneracy of the eigenmodes with respect to the radial order $n$ (cf. Unno et al. 1989).

Acknowledgments. This work was supported by the Grant-in-Aid for Scientific Research of the Ministry of Education, Culture, Sports, Science and Technology (No. 12047208) as well as the Japan Society for the Promotion of Science (Grant No. P-99748).

\section{References}

Ascher, U.M., Mattheij, R.M.M., Russell, R.D. 1995, Numerical Solution of Boundary Value Problems for Ordinary Differential Equations (Philadelphia: SIAM)

Christensen-Dalsgaard, J., Mullan, D.J. 1994, MNRAS, 270, 921

Gautschy, A., Glatzel, W. 1990a, MNRAS, 245, 154

Gautschy, A., Glatzel, W. 1990b, MNRAS, 245, 597

Glatzel, W., Gautschy, A. 1992, MNRAS, 256, 209

Lopes, I.P. 2001, A\&A, 373, 916

Unno, W., Osaki, Y., Ando, H., Saio, H., Shibahashi, H. 1989, Nonradial Oscillations of Stars, Second Edition (Tokyo: University of Tokyo Press) 